\title{
Análise da Probabilidade de Interrupção para Sistemas MIMO Cooperativo
}

\author{
Edgar B. Souza, Robson D. Vieira e Paulo H. P. Carvalho
}

\begin{abstract}
Resumo-A cooperação entre estações base num sistema de comunicação sem fio com múltiplas antenas é vista como uma das possíveis soluções para reduzir a interferência intercelular. Dentro desse contexto, este artigo apresenta uma análise de desempenho do enlace de descida de um sistema celular em que as estações base com múltiplas antenas transmitem ao mesmo tempo informações aos terminais dos usuários, que têm apenas uma antena. A probabilidade de interrupção do sistema é avaliada por meio de simulações e são estudadas as condições nas quais o esquema de cooperação proporciona ganhos mais significativos.
\end{abstract}

Palavras-Chave-MIMO cooperativo, probabilidade de interrupção.

Abstract-Cooperation between base stations in wireless communication systems with multiple antennas is considered as a candidate solution to reduce intercell interference. This paper presents the downlink performance analysis in cellular systems where multi-antenna base stations communicate with a couple of single-antenna terminals simultaneously. The system outage probability is evaluated with simulations and we study the conditions under which the cooperation between base stations provides higher gains.

Keywords-Cooperative MIMO, outage probability.

\section{INTRODUÇÃO}

Os sistemas MIMO (do inglês, multiple-input multipleoutput) têm sido um dos temas mais pesquisados no campo das comunicações sem fio durante os últimos anos. Isto se deve ao fato das antenas adicionais proporcionarem um grande aumento da eficiência espectral do sistema quando comparado ao desempenho de sistemas com apenas uma antena. Contudo, os impressionantes ganhos de multiplexação observados em enlaces ponto-a-ponto não estão presentes em enlaces ponto-amultiponto devido aos altos níveis de interferência do sistema.

A melhora do desempenho de sistemas ponto-a-multiponto constitui um grande desafio, que vem ganhando cada vez mais importância à medida em que são definidos os requisitos e especificações técnicas das futuras tecnologias de sistemas móveis celulares. Tecnologias como LTE e WiMAX preveem o emprego de múltiplas antenas de transmissão e recepção como parte do pacote de soluções técnicas que viabilizem serviços com elevadas taxas de transmissão e baixa latência. E para reduzir a interferência intercelular no enlace de descida, existem diversas propostas baseadas na cooperação entre estações base, também conhecida na literatura como processamento de sinal em múltiplas celulas [1], [2], [3].

Edgar B. Souza e Robson D. Vieira, Instituto Nokia de Tecnologia, Brasil. E-mails: \{edgar.souza, robson.domingos\}@indt.org.br. Paulo H.P. Carvalho, Departamento de Engenharia Elétrica, Universidade de Brasília, Brasil. Email: paulo@ene.unb.br
De fato, quando uma rede celular opera de maneira coordenada, o enlace de descida se resume a um canal de broadcast MIMO clássico [4] com restrições de potência por antena. Neste caso, a melhor estratégia para maximizar a capacidade total do sistema é a aplicação da técnica de codificação dirty paper, que consiste em realizar a formação de feixes com MMSE (do inglês, minimum mean square error) combinada ao precancelamento da interferência [5]. Apesar da utilização desta estratégia em sistemas reais ainda não ser possível devido a limitações técnicas, os resultados teóricos têm sido analisados como referência de desempenho para estratégias sub-ótimas.

Um dos requisitos básicos para a maioria dos modelos de cooperação propostos é o conhecimento perfeito do canal tanto nos terminais quanto nas estações base e até mesmo em outros elementos do sistema. Logo, é necessário que as informações a respeito dos canais, normalmente disponíveis nos terminais, sejam enviadas às estações base e, eventualmente, a outros elementos da rede. Em condições reais, tais informações não serão perfeitas devido a erros de estimação, sequências de treinamento não-ideais, atraso no canal de retorno, etc. Considerando alguns destes efeitos, os autores em [6] fazem um abrangente estudo da otimização do enlace de descida cooperativo e analisam a alocação ótima de recursos em diferentes cenários.

O trabalho apresentado em [7], por sua vez, propõe uma estratégia de alocação de potência que minimiza a probabilidade de interrupção de sistemas celulares cooperativos baseada apenas no conhecimento das estatísticas dos canais. Quanto a esse aspecto, esta proposta é mais realista, uma vez que o esquema de cooperação proposto requer uma capacidade extra menor para o canal de retorno. O trabalho contempla apenas sistemas com uma única portadora e há em [8] uma proposta de estratégia de alocação de potência para sistemas com múltiplas portadoras.

O estudo que será mostrado logo a seguir é uma extensão da análise de desempenho para o modelo apresentado em [7]. São realizadas simulações com o intuito de avaliar o comportamento do esquema de cooperação diante de um modelo realista para o canal sem fio. Além disso, são feitas algumas análises para identificar as condições nas quais o esquema provê ganhos mais significativos e o principal critério de avaliação do desempenho do sistema é a probabilidade de interrupção. Sendo assim, o artigo desenvolve-se da seguinte maneira: na seção II é revisto o modelo de sistema proposto em [7], assim como a alocação ótima de potência que minimiza a probabilidade de interrupção em um canal teórico; a seção III apresenta os detalhes do cenário de estudo e os parâmetros de 
simulação; por fim, as seções IV e V apresentam, respectivamente, a análise dos principais resultados e as considerações finais deste trabalho.

\section{Modelo do Sistema}

Será considerado o modelo em que $B$ estações base, que estão conectadas a uma estação central, se comunicam simultaneamente com $K$ terminais móveis. Este modelo de enlace de descida está ilustrado na Figura 1 para a situação específica na qual há apenas duas estações base e dois terminais.

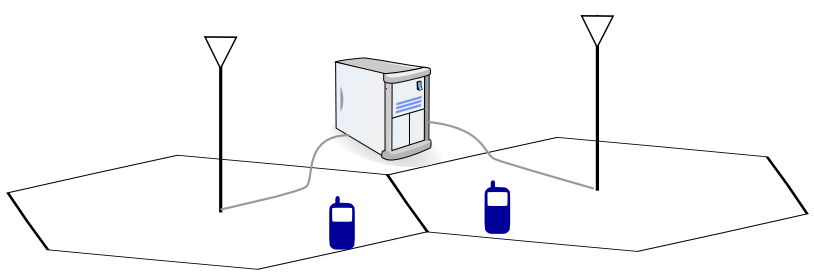

Fig. 1. Modelo do sistema

A transmissão dos dados da estação central até os terminais é caracterizada por duas etapas básicas:

1) A estação central gera as mensagens destinadas aos $K$ terminais e as encaminha para as estações base;

2) Cada estação base codifica as mensagens e as envia aos terminais de acordo com uma estratégia de alocação de potência pré-definida.

Para este modelo, deseja-se estabelecer um esquema de cooperação entre as estações base para alocar a potência disponível com o objetivo de minimizar a probabilidade de interrupção do sistema. Nessas condições, são estudados em [7] três cenários que diferem quanto ao conhecimento da estação central sobre os canais dos terminais. Os cenários são os seguintes:

- Conhecimento de todas as realizações dos canais;

- Conhecimento das estatísticas dos canais;

- Nenhum conhecimento dos canais.

Nos três cenários descritos acima assume-se que os terminais são capazes de estimar os canais de maneira perfeita e que as estações base sempre têm conhecimento total das realizações dos canais de todos os terminais. Este trabalho foca na análise de desempenho apenas do terceiro cenário.

Considera-se que cada estação base tem $M$ antenas, que cada um dos $K$ terminais tem apenas uma antena e que $K \leq M$. Além disso, não há nenhum enlace direto entre as estações base e, portanto, a comunicação entre elas é feita sempre com o intermédio da estação central. Tal comunicação ocorre através de enlaces ditos perfeitos, isto é, não há nem erros nem atrasos e a capacidade de tais enlaces é muito maior do que a capacidade mínima necessária para que a transmissão dos dados seja realizada com sucesso. Os canais sem fio $\mathbf{h}_{b k}$ entre a estação base $b$ e o terminal móvel $k$ não são seletivos em frequência e são modelados por variáveis aleatórias mutualmente independentes que seguem a distribuição normal complexa $\mathcal{N}_{\mathbb{C}}\left(\mathbf{0}, \sigma_{b k}^{2} \mathbf{I}_{M}\right)$.

Nas condições descritas acima, a estação central tem as mensagens $m_{1}, m_{2}, \ldots, m_{K}$ que devem ser enviadas aos terminais. Cada mensagem $m_{k}$ é dividida em $B$ partes $m_{1 k}, m_{2 k}, \ldots, m_{2 k}$ que, em seguida, são encaminhadas às respectivas estações base. Sendo assim, a estação base $b$ recebe da estação central as mensagens $m_{b 1}, m_{b 2}, \ldots, m_{b K}$. Considerando que a estação base $b$ poderia aplicar alguma técnica de codificação de canal, as mensagens $m_{b 1}, m_{b 2}, \ldots, m_{b K}$ seriam codificadas nos símbolos $s_{b 1}, s_{b 2}, \ldots, s_{b K}$ de maneira independente.

Neste modelo, a estação base $b$ aplica de maneira ideal uma técnica de pré-codificação linear, como, por exemplo, o zeroforcing transmit beamforming, nos símbolos $s_{b 1}, s_{b 2}, \ldots, s_{b K}$ com $\left|s_{b k}\right|^{2}=1$. Assim, o sinal de transmissão pode ser representado pela seguinte expressão:

$$
\mathbf{x}_{b}=\sum_{k=1}^{K} \sqrt{p_{b k}} \mathbf{g}_{b k} s_{b k}
$$

onde $\mathbf{g}_{b k}$ tem dimensão $M \times 1$ e representa o vetor de précodificação. Considera-se uma restrição de potência tal que o sinal transmitido pela $b$-ésima estação base tenha potência máxima igual a $P_{b}$

$$
\operatorname{tr}\left(\mathbb{E}\left[\mathbf{x}_{b} \mathbf{x}_{b}^{H}\right]\right) \leq P_{b}
$$

que pode ser simplificada assumindo que os vetores de précodificação são unitários, ou seja, $\left|\mathbf{g}_{b k}\right|^{2}=1$ para quaisquer $b$ e $k$ :

$$
\sum_{k=1}^{K} p_{b k} \leq P_{b}
$$

Como o vetor de pré-codificação $\mathbf{g}_{b k}$ é independente de $\mathbf{h}_{b k}$ e ortogonal a $\mathbf{h}_{b j}$ para $j \neq k$, então o sinal recebido pelo $k$ ésimo terminal é:

$$
\begin{aligned}
y_{k} & =\underbrace{\sum_{b=1}^{B} \sqrt{p_{b k}} \mathbf{h}_{b k}^{H} \mathbf{g}_{b k} s_{b k}}_{\text {sinal }}+\underbrace{\sum_{b=1}^{B} \mathbf{h}_{b k}^{H} \sum_{j \neq k}^{K} \sqrt{p_{b j}} \mathbf{g}_{b j} s_{b j}}_{\text {interferência }}+w_{k} \\
& =\sum_{b=1}^{B} \sqrt{p_{b k}} a_{b k} s_{b k}+w_{k}
\end{aligned}
$$

onde $a_{b k}=\mathbf{h}_{b k}^{H} \mathbf{g}_{b k}$ representa o ganho do canal entre a estação base $b$ e o terminal $k, w_{k}$ modela o ruído branco Gaussiano de média nula e variância unitária. Supõe-se que os terminais são capazes de realizar a detecção do tipo SIC (do inglês, successive interference cancelation) para receberem corretamente os símbolos que são enviados pelas estações base. Assim, a capacidade do $k$-ésimo terminal é:

$$
R_{k}=\log \left(1+\sum_{b=1}^{B}\left|a_{b k}\right|^{2} p_{b k}\right)
$$

em que $\left|a_{b k}\right|^{2}$ é uma variável aleatória que segue a distribuição chi-quadrado com $2(M-K+1)$ graus de liberdade [7]. A região de capacidade determinística do sistema para uma certa realização do canal a e para uma certa alocação de potência p é dada pela seguinte expressão:

$$
\mathcal{R}(\mathbf{a}, \mathbf{p})=\left\{\mathbf{R} \in \mathbb{R}_{+}^{K} \mid R_{k} \leq \log \left(1+\sum_{b=1}^{B}\left|a_{b k}\right|^{2} p_{b k}\right)\right\}
$$


e a região de capacidade considerando que as estações base têm as potências máximas limitadas a $\left(P_{1}, P_{2}, \ldots, P_{B}\right)$ será igual a:

$$
\mathcal{C}(\mathbf{a}, \mathbf{P})=\bigcup_{\sum_{k=1}^{K} p_{b k} \leq P_{b}} \mathcal{R}(\mathbf{a}, \mathbf{p}) .
$$

A alocação ótima de potência que minimiza a probabilidade de interrupção deste modelo de sistema é proposta em [7] para o cenário em que a estação central conhece todas as realizações dos canais e, também, para o cenário em que ela conhece apenas as estatísticas dos canais. Aqui é considerado somente o cenário no qual não há nenhuma informação $a$ priori que possa ajudar na alocação dos potência. Neste caso a solução é dividir toda a potência disponível igualmente entre os terminais. Assume-se que a potência máxima de cada uma das estações base é igual a $P$, que $p \triangleq p_{b k}=P / K$ e que $\sigma_{b k}=1$ para todos os enlaces. Isso implica em:

$$
\begin{aligned}
\Delta_{k} & =\sum_{b=1}^{B}\left|a_{b k}\right|^{2} p_{b k} \\
& =p \sum_{b=1}^{B}\left|a_{b k}\right|^{2} \triangleq \Delta_{k}^{e}
\end{aligned}
$$

onde $\Delta_{k}^{e}$ é uma variável aleatória de distribuição chi-quadrado com $4(M-K+1)$ graus de liberdade e sua função de distribuição cumulativa $F_{\Delta_{k}^{e}}(y)$ pode ser encontrada na literatura [9].

Assim, a probabilidade de interrupção teórica do sistema é igual a:

$$
P_{\text {out }}(\boldsymbol{\gamma}, \mathbf{p})=1-\prod_{k=1}^{K}\left(1-F_{\Delta_{k}^{e}}\left(c_{k}\right)\right),
$$

onde $c_{k}=2^{\gamma_{k}}-1$.

\section{CEnÁRio de Simulação}

O cenário básico adotado nas simulações é constituído por duas células, cada uma delas com uma estação base, e por diversos terminais que estão uniformemente distribuídos nas células. As estações base transmitem seus respectivos sinais simultaneamente a apenas dois terminais, que são escolhidos aleatoriamente. O modelo de canal adotado é baseado na soma de raios e está descrito em [10].

Existem, basicamente, dois modos de transmissão do sistema. No modo normal, cada estação base transmite sinal para apenas um terminal realizando a multiplexação espacial com duas antenas de transmissão e uma de recepção. No modo cooperativo, a transmissão é realizada da maneira descrita na seção II. Para a decisão do modo de transmissão do sistema, define-se a função $r:[0,1] \rightarrow \mathbb{R}^{+}$dada pela expressão:

$$
r(\xi)=(1-\xi) r_{c}
$$

onde $r_{c}$ é o raio da célula. As duas estações base operam em modo cooperativo se e somente se os dois terminais selecionados estiverem dentro da região sombreada da Figura 2. O tamanho da região sombreada é controlada pela variável $\xi$ da equação (10): quando $\xi=0$ as estações base operam no modo normal; quando $\xi=1$ as estações base operam no modo cooperativo independentemente da posição dos terminais; para os demais valores de $\xi$ é possível controlar o tamanho da região sombreada para que o modo cooperativo seja habilitado apenas para os terminais que estiverem mais próximos das bordas e, consequentemente, para que o modo normal seja habilitado apenas para os demais terminais que estiverem mais próximos do centro da célula.

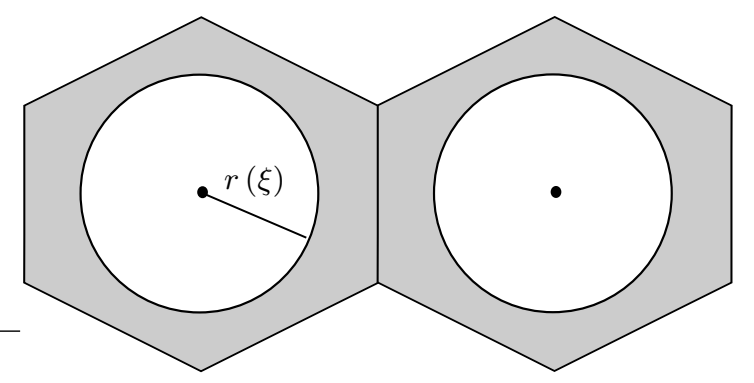

Fig. 2. Cenário de simulação

Os parâmetros utilizados nas simulações são listados na tabela a seguir. Os parâmetros que não são explicitamente citados, tais como espalhamento angular, distribuição de atrasos, distância de correlação e outros, têm seus respectivos valores definidos em [10] e [11].

TABELA I

PARÂMETROS DE SiMULAÇÃo

\begin{tabular}{||l|l||}
\hline \hline Parâmetros Gerais & Valor \\
\hline Número de estações base & 2 \\
\hline Distância entre as estações base & 1500 metros \\
\hline Distribuição dos terminais & uniforme \\
\hline Frequência central & $2.5 \mathrm{GHz}$ \\
\hline Distância mínima entre estação base e terminal & 35 metros \\
\hline Condição de propagação & sem linha de visada \\
\hline Desvio-padrão desvanecimento lento & $8 \mathrm{~dB}$ \\
\hline Largura de banda & $12 \mathrm{kHz}$ \\
\hline \hline Parâmetros da Estação Base & Valor \\
\hline Número de setores & 1 \\
\hline Altura & $35 \mathrm{metros}$ \\
\hline Tipo de antena & omnidirecional \\
\hline Número de antenas & 2 \\
\hline Espaçamento entre as antenas & $5 \lambda$ \\
\hline \hline Parâmetros do Terminal & Valor \\
\hline Altura & $1.5 \mathrm{metros}$ \\
\hline Tipo de antena & omnidirecional \\
\hline Número de antenas & 1 \\
\hline Velocidade & $3 \mathrm{~km} / \mathrm{h}$ \\
\hline Figura de ruído & $7 \mathrm{~dB}$ \\
\hline \hline
\end{tabular}

\section{Resultados}

As Figuras 3 e 4 mostram a probabilidade de interrupção média do sistema e de cada uma das células separadamente em função da potência de transmissão considerando $\xi=1$. Quando a capacidade requerida pelos terminais é $\gamma=[1,1]$ bits/s/Hz, as células apresentam valores muito próximos para a probabilidade de interrupção. No caso em que $\gamma=[1,3]$ bits/s/Hz, o terminal que requer maior capacidade está sempre na célula 2 e, nesta célula, a probabilidade de interrupção é bem maior que na célula 1 . Por isso, o desempenho do sistema é muito próximo do desempenho da célula 2 . 


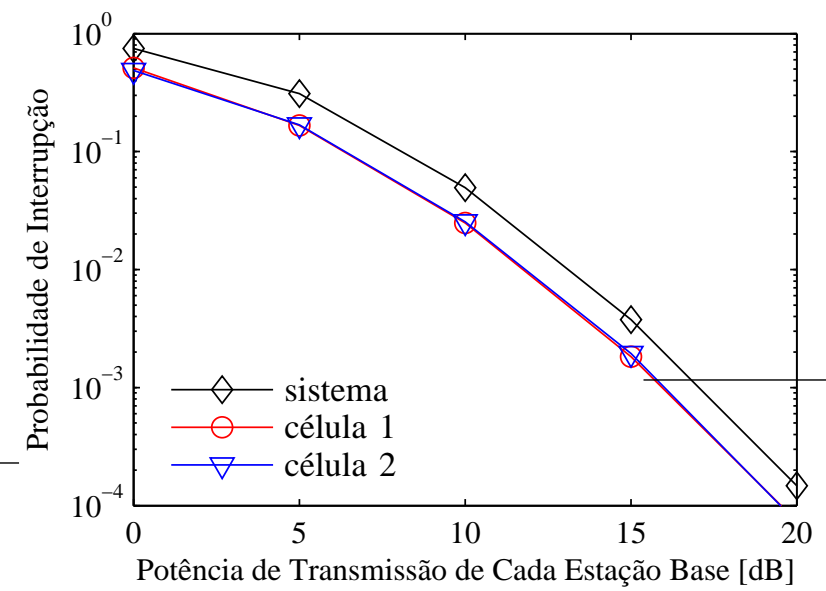

Fig. 3. Probabilidade de interrupção em função da potência de transmissão para $\gamma=[1,1]$ bits/s/Hz

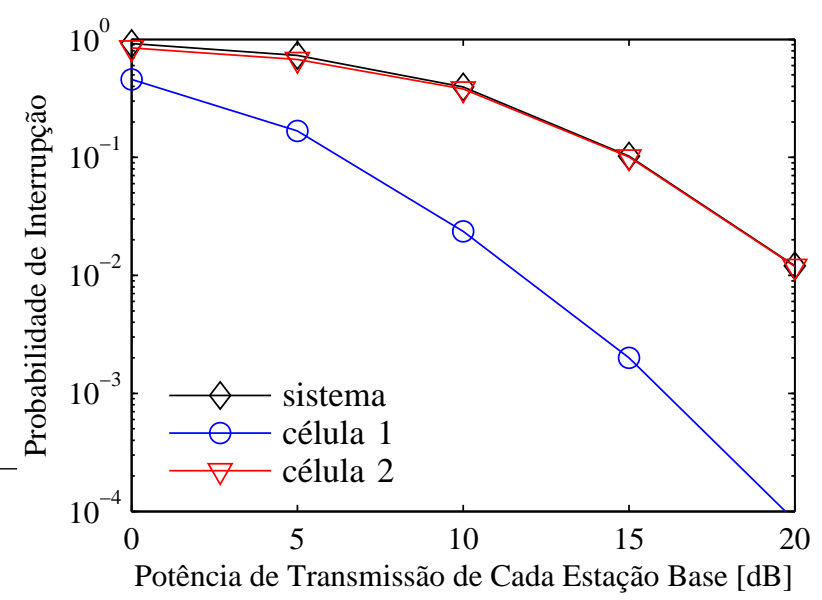

Fig. 4. Probabilidade de interrupção em função da potência de transmissão para $\gamma=[1,3] \mathrm{bits} / \mathrm{s} / \mathrm{Hz}$

Na Figura 5 é apresentado o desempenho do sistema para $\gamma=[1,1]$ bits/s/Hz e alguns valores de $\xi$. Nestas simulações considera-se que a capacidade requerida por cada terminal é igual a $1 \mathrm{bit} / \mathrm{s} / \mathrm{Hz}$. Observa-se que o melhor desempenho do sistema foi obtido quando $\xi=1$, pois nesta configuração o modo cooperativo proporciona ganhos mais significativos não apenas para os terminais que estão na região central do cenário, mas também para os que estão nas bordas do cenário. Logo, todos os terminais podem se beneficiar bastante dos ganhos de multiplexação espacial providos pelas duas estações base. Além destes aspectos, observa-se que é melhor que o sistema opere no modo normal quando a potência de transmissão é baixa. Nessas condições, o ganho de multiplexação do esquema de cooperação é insignificante e, dessa maneira, o sistema poderia reduzir a carga dos canais de retorno. Contudo, à medida em que a potência de transmissão aumenta, torna-se mais vantajoso habilitar a cooperação entre as estações base.

Os resultados da Figura 6 mostram como a distância entre as estações base e os terminais influencia na probabilidade de interrupção das células. Nesta figura são apresentados resultados para o modo cooperativo e para o modo normal

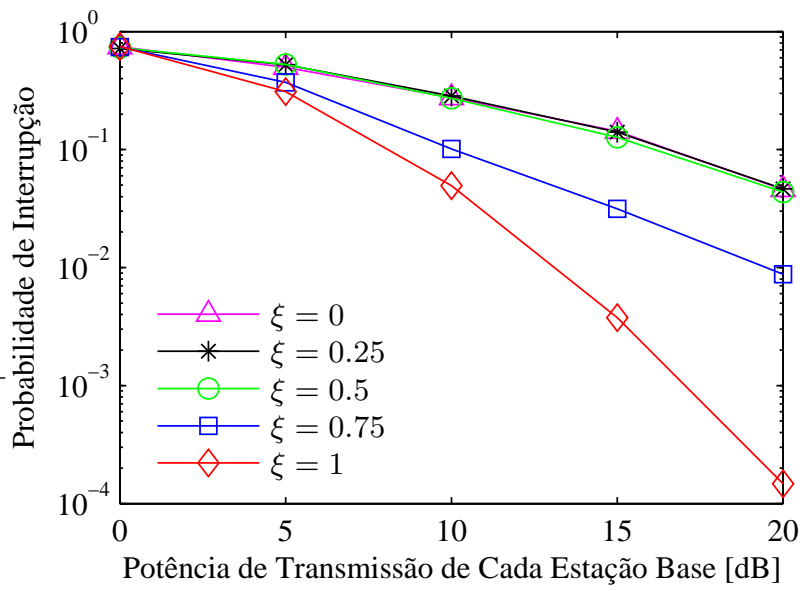

Fig. 5. Probabilidade de interrupção em função da potência de transmissão para $\gamma=[1,1]$ bits $/ \mathrm{s} / \mathrm{Hz}$

nos quais consideram-se três valores diferentes para a potência de transmissão das estações base. Observa-se nos três casos que os ganhos do modo cooperativo em relação ao modo normal diminuem à medida que das distâncias aumentam. Nestes gráficos fica evidente que os terminais que estão nas bordas das células e experimentam condições de propagação ruins se beneficiam menos do esquema de cooperação do que os outros terminais que estão no centro da célula. Por isso, o modo cooperativo não apresenta ganhos expressivos para $\xi<0.5$ na faixa de potência mostrada na Figura 5.

Supondo que os terminais deste sistema de comunicação sejam requisitados a operar com probabilidade de interrupção inferior a, por exemplo, $10^{-3}$, então os dados da Figura 6 poderiam auxiliar na escolha do modo de operação e da potência de transmissão das estações base. Neste exemplo, seria necessário que a potência de transmissão fosse igual a $20 \mathrm{~dB}$ para que o sistema no modo normal atendesse aos requisitos somente dos terminais com distâncias inferiores a 350 metros de suas respectivas estações base. Contudo, no modo cooperativo seria possível atender estes mesmos terminais com a potência de transmissão igual a apenas 10 dB. Por outro lado, se a potência de transmissão fosse mantida em $20 \mathrm{~dB}$, todos os terminais do sistema estariam operando abaixo do limite máximo da probabilidade de interrupção.

O mapa das Figuras 7 e 8 mostra a probabilidade de interrupção medida em cada parte do cenário de simulação para o caso $\xi=1$. As estações base estão posicionadas nas coordenadas $\left(x_{1}, y_{1}\right)=(750,750)$ e $\left(x_{2}, y_{2}\right)=(2250,750)$ e a potência de transmissão é igual a $10 \mathrm{~dB}$. Os pontos em azul indicam os locais em que foram medidos os menores valores e os pontos em vermelho indicam os valores mais altos para a probabilidade de interrupção. A Figura 7, confirma que o desempenho médio das células é bem parecido para o caso em que a capacidade requerida é $\gamma=[1,1] \mathrm{bits} / \mathrm{s} / \mathrm{Hz}$. A Figura 8 , por sua vez, mostra o mapa $\gamma=[1,3] \mathrm{bits} / \mathrm{s} / \mathrm{Hz}$. Nela, a região $x>1500$ metros apresenta um desempenho médio pior pois corresponde à área de cobertura da célula 2 e o terminal que requer maior capacidade sempre é posicionado dentro desta célula. 


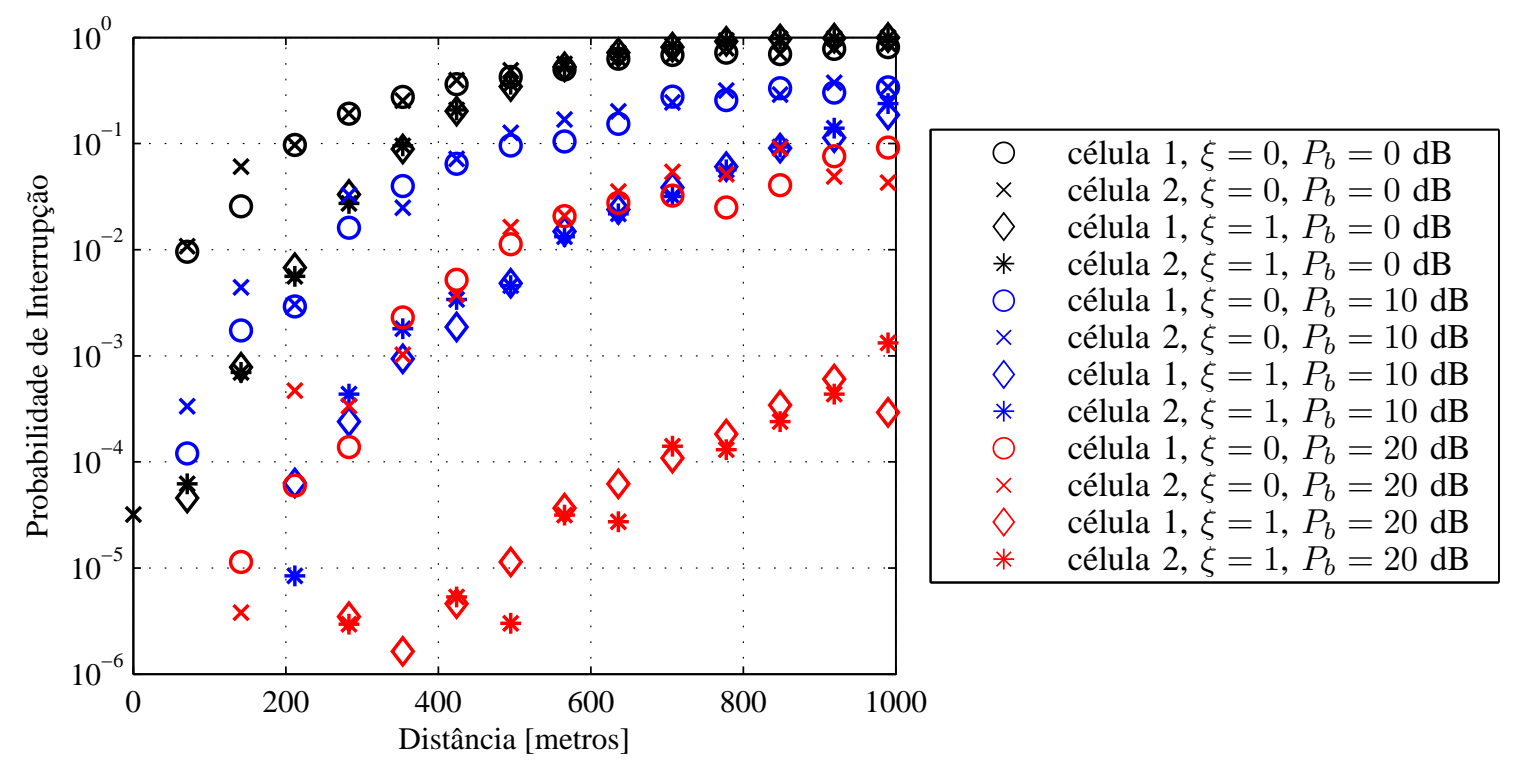

Fig. 6. Probabilidade de interrupção em função da distância para alguns valores de potência de transmissão
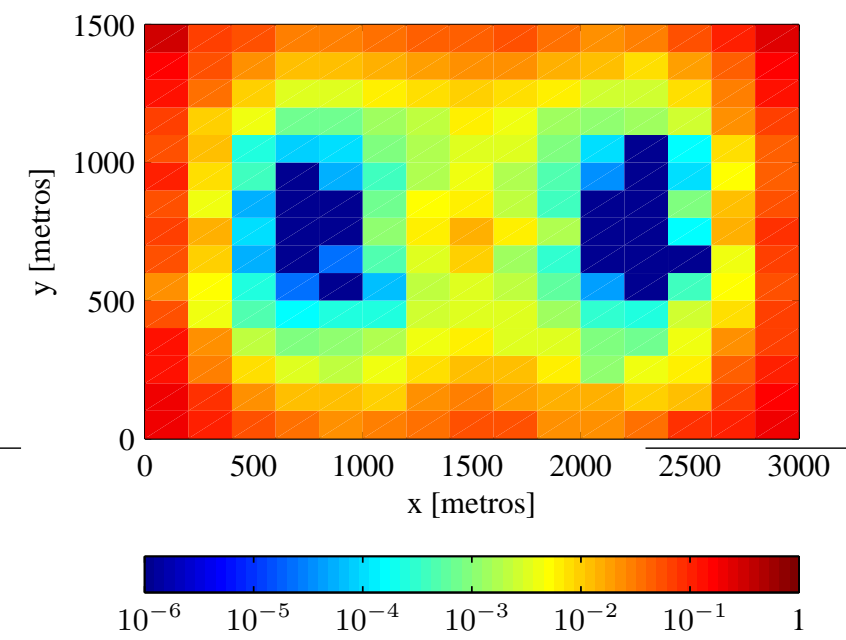

Fig. 7. Mapa da probabilidade de interrupção para $\gamma=[1,1]$ bits/s/Hz

\section{CONClusões}

Os resultados apresentados na seção anterior fornecem as primeiras diretrizes de aplicação do modelo de cooperação proposto por [7] em sistemas reais. De modo geral, pode-se afirmar que os ganhos do modo de transmissão cooperativo são mais significativos quando a potência de transmissão das estações base é maior. Por este motivo, os terminais mais próximos das estações base experimentam ganhos maiores em todas as situações analisadas, enquanto que o desempenho dos terminais mais próximos das bordas das células melhora apenas quando a potência de transmissão das estações base é maior.

Para um sistema de comunicação que não é limitado pela capacidade dos canais de retorno, a melhor estratégia con-
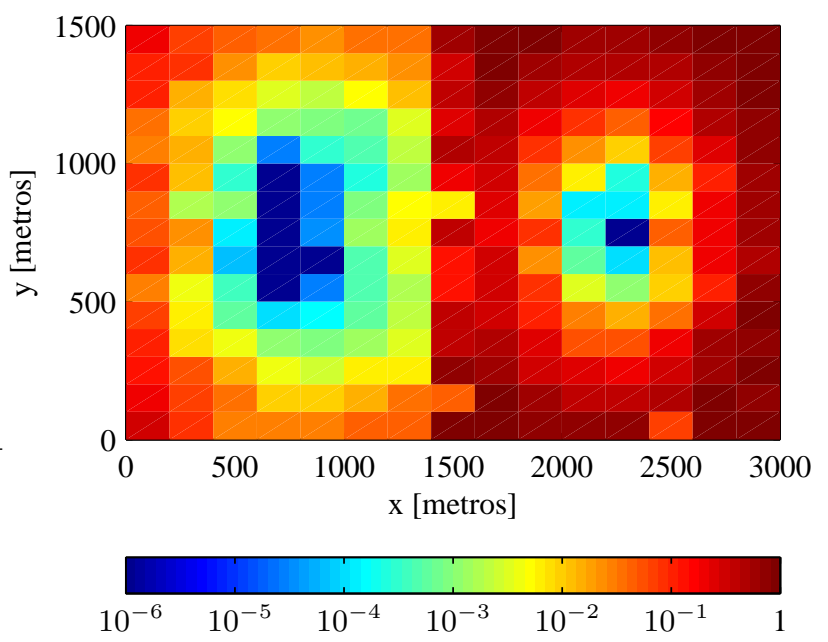

Fig. 8. Mapa da probabilidade de interrupção $\gamma=[1,3]$ bits/s/Hz

siste em ativar o modo cooperativo para todos os terminais. Contudo, se os canais de retorno forem limitados, torna-se necessário definir critérios mais complexos para a seleção do modo de transmissão. Tais critérios poderiam considerar não apenas a posição dos terminais em relação à estação base, mas também a capacidade requerida, por exemplo.

Conforme mencionado na seção III, o modelo original considera um canal ideal e seriam necessárias algumas alterações para explorar as características específicas do modelo de canal que foi empregado aqui.

A extensão natural deste trabalho é a análise da probabilidade de interrupção de um sistema com múltiplas estações base transmitindo ao mesmo tempo para, eventualmente, múltiplos terminais. Certamente este seria um cenário de 
simulação bem mais complexo do que o que foi considerado aqui e talvez seja necessário analisar uma quantidade bem maior de parâmetros para otimizar a escolha dos modos de operação do sistema.

Outro ponto relevante a ser destacado está relacionado aos requisitos para a implementação do modelo de cooperação. Este trabalho considera que o sistema de comunicação está sob condições ideais quanto à estimação do canal, enlaces de retorno, sincronismo de transmissão e de recepção. Estes fatores podem alterar significativamente o desempenho do sistema e o impacto deles ainda é um tópico a ser investigado.

Os aspectos descritos anteriormente são partes integrantes do processo de estudo e validação do modelo. A avaliação do modelo por meio de simulações em sistemas de comunicação com condições mais realistas é um passo muito importante para que ele se consolide como uma solução prática de redução da interferência inter-celular.

\section{AGRADECIMENTOS}

Este trabalho foi parcialmente financiado pelo Instituto Nokia de Tecnologia (INdT), através do Projeto WiSIL, e pelo CNPq, através do projeto WiPA, processo no. 554047/2006-3.

\section{REFERÊNCIAS}

[1] J. G. Andrews, W. Choi, and R. W. H. Jr., "Overcoming interference in spatial multiplexing MIMO cellular networks," IEEE Wireless Communications, vol. 14, no. 6, pp. 95-104, Dec 2007.

[2] H. Zhang and H. Dai, "Cochannel interference mitigation and cooperative processing in downlink multicell multiuser MIMO networks," EURASIP Journal on Wireless Communications and Networking, vol. 2004, no. 2, pp. 222-235, 2004.

[3] S. Jing, D. Tse, J. Soriaga, J. Hou, J. Smee, and R. Padovani, "Multicell downlink capacity with coordinated processing," EURASIP Journal on Wireless Communications and Networking, vol. 2008, 2008.

[4] D. Tse and P. Viswanath, Fundamentals of wireless communication. New York, NY, USA: Cambridge University Press, 2005.

[5] G. Caire and S. Shamai, "On the achievable throughput of a multiantenna Gaussian broadcast channel," IEEE Transactions on Information Theory, vol. 49, no. 7, pp. 1691-1706, 2003.

[6] P. Marsch and G. Fettweis, "On multicell cooperative transmission in backhaul-constrained cellular systems," Annales des Télécommunications, vol. 63, no. 5-6, pp. 253-269, 2008.

[7] M. Kobayashi, M. Debbah, and J.-C. Belfiore, "Outage efficient strategies for network MIMO with partial CSIT," in IEEE International Symposium on Information Theory, Jun 2009.

[8] E. B. Souza, R. D. Vieira, and P. H. P. Carvalho, "Outage probability for multicarrier cooperative MIMO with statistical channel knowledge," in IEEE Vehicular Technology Conference, VTC-Fall 2009, Anchorage, Alaska, USA, Sep 2009.

[9] A. Papoulis and S. U. Pillai, Probability, random variables, and stochastic processes, 4th ed. New York, NY, USA: McGraw-Hill, 2002.

[10] IST-WINNER II, "D1.1.2 WINNER II channel models," [online] Available: https://www.ist-winner.org/, Sep 2007.

[11] _ "D5.4 Final report on link level and system level channel models," [online] Available: https://www.ist-winner.org/, Nov 2005. 